\title{
ESPACIOS Y ARQUITECTURAS ESCOLARES. MATERIALIDADES, REGLAMENTACIONES Y CUERPOS EN EL PASADO Y EN EL PRESENTE
}

\author{
CARLOS ORTEGA IBARRA ${ }^{1}$ \\ ORCID: https://orcid.org/0000-0003-3916-9543 \\ LAURA CHÁZARO GARCÍA ${ }^{2}$ \\ ORCID: https://orcid.org/0000-0003-0154-5662
}

La escuela, esa institución que nació con y contribuyó a formar el mundo moderno, cerró sus puertas para contener la propagación de la Covid-19. Muy poco antes nos reunimos, un grupo disciplinariamente plural, conformado principalmente por historiadoras, pedagogas, sociólogas, antropólogas, arquitectos y comunicólogos de Brasil, México, Colombia y Argentina. Primero en la Ciudad de México (México), en junio de 2018 y, luego en agosto de 2019, en Córdoba (Argentina). Nos reunió el interés por reflexionar sobre los espacios y las arquitecturas en la escuela, sin imaginar que en 2020 una pandemia trastocaría el orden mundial. Entonces, las clases en línea, la educación remota, la escuela en casa fueron estrategias adoptadas por la mayoría de los gobiernos como una forma segura de continuar con los procesos de la educación escolarizada. Aunque ya habían sido ensayadas e implantadas en distintos niveles educativos, e incluso podrían recordarnos formas pretéritas de la educación a distancia mediante el servicio postal y la radiodifusión, la propagación acelerada de la enfermedad impuso la incorporación generalizada de estas estrategias sin estar exentas de contradicciones ni resistencias.

Paulatinamente, las escuelas han tomado formas nuevas, los miembros de las comunidades escolares han aprendido a relacionarse virtualmente, los espacios domésticos -ahora exhibidos públicamente- han sido reorganizados y re-escenificados para albergar funciones y actividades que se pensaba correspondían únicamente a la escuela, mientras tanto, los edificios escolares lucen vacíos poniendo al espacio en el centro de las preocupaciones de políticos y especialistas: se pasa de reflexiones sobre el espacio virtual a cuestionar cómo reacomodar los espacios físicos, quizá mediante modalidades que combinen el modo presencial con el virtual en un posible regreso escalonado a las escuelas. También se plantea la obsolescencia de una escuela amurallada cuya debilidad fue puesta en evidencia por la pandemia, y se propone la construcción de novedosas arquitecturas escolares que garanticen la salud de sus habitantes y sean compatibles con las tendencias educativas de la época digital. Si bien la arquitectura y los espacios escolares, o espacios para la enseñanza, no son un tema nuevo, la pandemia nos ha mostrado su centralidad,

\footnotetext{
${ }^{1}$ Dirección de Difusión de Ciencia y Tecnología del Instituto Politécnico Nacional. Ciudad de México, México. $<$ cortegai@ipn.mx $>$

2 Departamento de Investigaciones Educativas del Centro de Investigación y de Estudios Avanzados del Instituto Politécnico Nacional. Ciudad de México, México. <chazaro@cinvestav.mx> Educação em Revista | Belo Horizonte|v.37|e100037|2021
} 
como tema, pero también como un enfoque fructífero, pues al menos desde hace treinta años se ha constituido en un campo prometedor para las investigaciones educativas en América Latina.

En estas nuevas situaciones, en tránsito y cuyo destino no podemos conocer con certeza, nos parece una gran oportunidad que Educação em Revista publique los trabajos presentados en el Primer Taller de Espacios y Arquitecturas en la Escuela, organizado por Laura Cházaro, Inés Dussel y Carlos Ortega en el Departamento de Investigaciones Educativas del Cinvestav en la ciudad de México en 2018. Este Dossier continua la reflexión iniciada en aquel momento y cuyos primeros resultados ya fueron publicados en el Anuario de Historia de la Educación [Vol. 20, número 1,2019]. En este número propusimos trabajos que piensan los espacios escolares, sus arquitecturas y su materialidad desde distintas perspectivas disciplinarias y conceptuales con el fin de proporcionar algunas pautas que nos permitan imaginar con todos los sentidos dispuestos el futuro de las escuelas.

En las investigaciones que se reúnen en este Dossier confluyen enfoques provenientes de la sociología, la antropología, la geografía, la pedagogía, la comunicación y la historia, y están dirigidos a desentrañar las múltiples implicaciones de la arquitectura y los espacios escolares en la cultura y la organización de la sociedad. Aquí ofrecemos algunos casos de Brasil, México y Argentina, los cuales ilustran un diálogo disciplinario, geográfica y temporalmente variado, tan necesario en las condiciones actuales de las escuelas. Podemos decir que los seis textos reunidos, plurales en enfoques y metodologías, forman un todo coherente por razones temáticas y analíticas. Una característica común de los artículos presentados es centrar los análisis en el espacio como una dimensión material de la vida escolar, sean las prácticas pedagógicas o las experiencias mismas de planear y habitar ese espacio como escolares, profesores, diseñadores, políticos y funcionarios públicos.

Vale la pena preguntarse ¿por qué el cuerpo, como representación y como performance, es tan central cuando hablamos del espacio escolar? Las políticas y las propias instituciones, a la hora de planear los espacios se topan de frente con los cuerpos, ya sean los cuerpos infantiles o de los profesores, como también los de los animales y con otras cosas u objetos. El espacio escolar supone, a un nivel muy general, a la arquitectura, a la materialidad de los inmuebles y a la escuela en su relación con los barrios y luego con las ciudades. Como lo muestra Henri Lefebvre, supone una dimensión relacional que implica disciplinar, dirigir, controlar, producir y recrear cuerpos. Estas condiciones suceden en espacios como los hospitales, los laboratorios o las escuelas, y de manera específica en los salones de clases.

Ya se trate de México, Brasil o Argentina, los autores coinciden en que el enfoque material supone un compromiso analítico que obliga a reflexionar seriamente sobre ¿cómo pensar la arquitectura-espacio escolar? Marcus Levy, Alejandra Castro y Carlos Ortega responden esta cuestión haciendo de las leyes objetos materiales de reflexión. Cada uno se pre-ocupa de reformas educativas a las que analizan como fuente (Levy) y como puesta en práctica de un cierto espacio (Castro y Ortega).

Marcus Levy, desde la perspectiva del historiador y basado en el modelo indiciario de Carlo Ginzburg (1986), sumerge a sus lectores en la central cuestión epistemológica sobre sus fuentes (el archivo). En Os regulamentos para a construção dos edifícios escolares públicos no Brasil: o exemplo do estado do Paraná na primeira metade do século, Levy se interroga cómo dar cuenta de la política de inversión del estado brasileño. Como otros historiadores, recurre a los códigos, reglamentos e instrucciones específicamente destinadas al funcionamiento de escuelas primarias en Paraná (Brasil), entre los años 1903-1938. Levy no quiere ahogar las voces de otros sujetos, pues además de las autoridades escolares, muestra cuánto esos materiales administrativos implican una multiplicidad de voces que es necesario sacar de la oscuridad interpretativa y poner a dialogar. El archivo es un cuerpo que, para escribir la historia de la arquitectura, supone un diálogo y no una mera colección de decretos. La escritura de la historia de la arquitectura exige según Levy, un camino indiciario y una metodología de interpretación que le permita evitar tanto los dejá vu como los abismos del anacronismo. Para esto último se vale de los cuatro niveles de interpretación 
utilizados por los sabios judíos del siglo XIII, conocido por el acróstico PaRDeS (פָרְרָד), con el que se revelan los sentidos profundos de las fuentes.

Con el mismo impulso que pretende robustecer la aproximación analítica de espacio y materialidad, Alejandra M. Castro nos entrega el artículo Espacialidade escolar, leituras de foco e deslocamentos na (des) construção de um objeto. Desde una perspectiva sociológica, sin dejar de apelar a la filosofía, la autora analiza la vinculación entre las políticas educativas y la espacialidad. Resultado de un proyecto más amplio, el artículo toma como campo empírico los textos políticos que expresan las políticas educativas para garantizar el derecho a la educación y la obligatoriedad de la escuela secundaria en Argentina. Se enfoca en la Ley de Educación Nacional Nº 26.206 (LEN) de 2006 que amplió a más años la obligatoriedad del nivel inicial, haciendo obligatoria la escuela secundaria e incrementando la carga horaria en la escuela primaria vía la jornada ampliada. Para dar cuenta del significado de la inclusión social, el artículo se propone elucidar "cómo y de qué maneras lo espacial se articula o desarticula con estos propósitos de la política educativa". Así, la autora comienza por convertir en pregunta algo que regularmente se asume como dado: ¿qué se está diciendo cuando se habla de espacio escolar? Para responder esta cuestión echa mano de las perspectivas antropológicas y sociológicas de Henri Lefebvre y Doreen Massey. El principal aporte de esta reflexión epistemológica es mostrar que el espacio escolar es una multiplicidad de posibilidades, desde la materialidad del edificio, la trayectoria y las distancias; pero también un dispositivo que produce cuerpos y sus sentidos, una institución y un lugar de socialización. Entre más dimensiones mostramos, como lo dice la autora, mayor es el desafío de construir su especificidad. En su trabajo empírico, justamente, lo que busca es resolver como lo político, en el caso de la ley de 2006, se implica, se vuelve acción inclusiva (performativa del cuerpo) en los espacios escolares. Lo que el artículo nos enseña es cuánto queda de las relaciones políticas que ordenan el espacio escolar, por más neutro, formal y abstracto que se presente ya sea como ley o en las formas de ocupación. El espacio es político y, en ese sentido, son contingentes e históricos sus significados, sus materialidades y las vivencias que lo posibilitan.

Carlos Ortega, como los dos autores anteriores, parte de decretos oficiales, proyectos arquitectónicos postrevolucionarios y experimentos para crear nuevos espacios escolares para obreros. El artículo Aprender a habitar a arquitetura escolar da nova ordem urbana (Distrito Federal, México, 1932) deconstruye el espacio echando mano de sus múltiples dimensiones: como parte de proyectos urbanos para la Ciudad de México, como arquitectura de una escuela y como lugar de enseñanza. Así Ortega pone en la misma arista de sus cuestionamientos: cuerpos, arquitectura, espacio público y urbano, y decretos y políticas educativas. Específicamente el artículo analiza cómo un grupo de técnicos y políticos, dirigidos por el arquitecto funcionalista Juan O'Gorman (1905-1982), participaron de su idea de crear un nuevo orden urbano, aprovechando la reforma del regímen político del Distrito Federal, capital del país, y buscar los medios para gobernar esa ciudad que no paraba de crecer. El autor enfatiza cómo la Escuela primaria Emiliano Zapata, construida en la Colonia Industrial S.A., no solo representa a la arquitectura funcionalista inspirada en Le Corbusier (1925). Analizando los restos de esa Escuela y la propia colonia el autor logra mostrar cuánto de la arquitectura técnica o funcional fue ante todo un proyecto político, un programa que pretendía reprogramar los cuerpos infantiles y de los profesores mediante una racionalidad moderna. El miedo al desorden del pueblo, ya mostrado durante la Revolución de 1910, no debía repetirse. Construir una escuela fue un acto técnico y político, que buscaba materializar un nuevo orden, el que favoreciera una racionalidad productivista, que transformara a las clases populares en obreros sanos. En el análisis de Levy la escritura de la historia exige al historiador dejar de considerar sus fuentes como inertes pruebas del pasado, y para saber de la arquitectura remite a las complejas gramáticas de las fuentes. El texto de Ortega nos muestra también que escribir historia además implica al cuerpo del historiador. Efectivamente, para aprender sobre los espacios de aprendizaje del siglo pasado, convierte a la ciudad en testimonio visitable, cognoscible. Así, el autor camina para descubrir en las calles de la ciudad de hoy las distintas huellas de las fuentes. El diseño urbano es el archivo y, como lo demuestra el autor, en 
sus diferentes vestigios se muestran los arreglos y proyectos políticos de la élite de técnicos y arquitectos de aquella época.

Los artículos de Rosalía Meníndez, Laura Cházaro y Daniel Hernández piensan el espacio escolar desde la compleja relación entre los objetos, los sujetos, los animales y las cosas. Si bien la institucionalidad importa, su existencia toma forma a través de las relaciones entre las cosas: ya sea en los salones de clases, en las tecnologías de comunicación o en los laboratorios.

Daniel Hernández ofrece un análisis del espacio poniendo en el centro la cuestión de cambio y novedad. Se pregunta ¿cómo llevar el salón de clases en sintonía con los cambios tecnológicos?, ¿cómo innovar los espacios escolares? Interesado en los fenómenos de lo que él llama "educomunicación", analiza y discute las posibles variaciones de la relación -comunicativaentre el profesor y los alumnos si cambiamos la disposición física de las cosas de los salones de clases, como sillas o pizarras. Estos cambios, según el autor, posibilitarían "métodos mayormente activos y participativos de enseñanza y aprendizaje." Desde la perspectiva de la comunicación, explora así distintos diseños de aula, como el "diseño activo" que promueve didácticas de aprendizaje por descubrimiento, exploración, experimentación, crítica y análisis; o el modelo de "zonas de aprendizaje" basado en la idea de romper con espacios o salones de clases uniformes. Basado en el "Aula del Futuro", proyecto desarrollado en el Instituto de Ciencias Aplicadas y Tecnología de la UNAM, el autor nos propone integrar las TIC's al proceso educativo y crear nuevos espacios colaborativos, más allá del salón de clases. Para Hernández, el espacio educativo tendría que traducirse en "arquitecturas de aprendizaje", donde las computadoras, pero también el mobiliario, las pizarras y las pedagogías puedan fomentar y trabajar las habilidades requeridas por los estudiantes para el siglo XXI. En esa perspectiva la tecnología no se reduce a la computadora o a la tableta, implica considerar los objetos y las cosas que, dentro y fuera de la escuela, la hacen funcionar.

Desde una perspectiva de la historia cultural de las ciencias, el texto de Laura Cházaro pretende iluminar la relación entre los espacios y el conocimiento. Intitulado Espaços de ensino clínico e experimental: hospitais e laboratórios na cidade do México, século 19 se enfoca en cómo circulan las materialidades, los cuerpos, las cosas y hasta los animales en la enseñanza de la medicina del siglo antepasado. Deconstruyendo las prácticas de los profesores y estudiantes de medicina, demuestra que los espacios de enseñanza no son unidimensionales, ni en representación, ni físicamente. La medicina, como otras disciplinas científicas, se enseñan en espacios y tiempos híbridos. Con su estudio de caso, la autora muestra cómo la enseñanza de la medicina se dio en espacios híbridos, como el hospital-escuela-laboratorio, pues la Escuela Nacional de Medicina no podría entenderse sin el espacio clínico y experimental del Hospital de San Andrés. Esos espacios, hoy opacados o tomados por separado, dan cuenta de cómo los cuerpos que se pretenden curar, así como los de los propios profesores y estudiantes, son creados y recreados. No solo por las exigencias de la disciplina médica sino también por las tensiones políticas que constituyen esos espacios, por ejemplo, la escuela versus el hospital, las jerarquías entre profesores y alumnos y hasta los debates teóricos entre experimentalistas y clínicos.

La cuestión de los cuerpos y los espacios de enseñanza son el tema central del artículo de Rosalía Meníndez, O espaço arquitetônico e o regulamento dos órgãos das crianças: As salas de aula, no fim do século XIX e com o início do século XX. Este artículo revela la compleja relación que establecen los cuerpos en el espacio escolar valiéndose de la reconstrucción de planos de escuelas "tipo" propuestos por médicos e higienistas, funcionarios del Ministerio de Instrucción Pública, entre fines del siglo XIX y principios del XX. En primer lugar, muestra cómo la configuración del espacio escolar "moderno" implicó el control de los cuerpos infantiles, cuestión demostrada en una amplia bibliografía. En segundo lugar, la autora pone de relevancia otras dimensiones que participan en la compleja co-construcción de cuerpos en edad escolar y espacios. Examinando diseños o recomendaciones para la construcción de edificios escolares, Meníndez nos hace ver cómo las cosas u objetos y los cuerpos, según el diseño, siguen, respetan o adoptan ciertas regulaciones y distribuciones. El mobiliario, los materiales escolares y los cuerpos se regulan, viven 
ciertas cercanías o distancias y densidades. A través de la representación de planos, el artículo de Rosalía Meníndez nos revela la importancia de las materialidades espaciales, o de cómo las cosas y los cuerpos conforman lo espacial.

En suma, el aporte más importante de los artículos aquí reunidos es doble. Por un lado, confirman la riqueza analítica y temáticas de lo que se ha llamado el enfoque o giro espacial. Pero también ofrecen reflexiones situadas, locales de esos enfoques teóricos. No solo porque los casos aquí presentados provienen de experiencias y situaciones en América Latina. Sobre todo, porque cada autor se implica en un diálogo, confrontación y réplica con sus referencias teóricas e inspiraciones metodológicas. Ya sea como historiadores, antropólogos, sociólogos o comunicólogos, cada uno ofrece, en su particularidad, una enorme riqueza temática y teórica para abordar ese giro espacial. Un segundo aporte del Dossier es su actualidad frente a nuestras experiencias por el Covid-19. Leídos desde la pandemia, cada texto ofrece ya una genealogía de cómo los cuerpos, las enfermedades y la tecnología, en suma, las cosas y sus arreglos espaciales, han tomado las actuales configuraciones en las escuelas.

El Dossier no hubiera sido posible sin la generosidad y colaboración de Inés Dussel, a quien le agradecemos su participación en la organización del Primer Taller de Espacios y Arquitecturas en la Escuela y su apoyo para completar este Dossier. También agradecemos a los evaluadores de Educaçã em Revista cuyos dictámenes contribuyeron a mejorar la calidad de los artículos que los lectores tienen ante sí. 